locally, and atropin given internally without result. As the patient complained of great pain and formication in the right angle of the mouth, the hydrobromate of hyoscine was given internally and a 5 per cent. solution of menthol locally with some relief. The hyperidrosis continued, however. The physiological relation between the facial tract and sweating was demonstrated by Adamkiewicz. Pathological observations on the relations of the trigeminus with sweating are very rare.

F. H. P.

\title{
PATHOLOGY OF THE NERVE PLEXUSES OF THE INTESTINES.
}

Bouome: Archivio delle Scienze Mediche. The author, after an extended series of researches, comes to the following conclusions :

I. Extirpation of the colliac ganglion, with partial destruction of the solar plexus, produces constantly in rabbits atrophy of intra-intestinal nervous plexus, accompanied with profound atrophy of the liver and spleen and general marasmus.

2. Atrophy of the intestinal plexus and general marasmus are more pronounced when there develop neuromata and fibro-neuromata after the extirpation of the colliac plexus.

3. There exists no relation between the nutritive activity of the intestinal plexuses and the muscular tone of the intestines.

4. Obstructing the circulation in the intestines produces throughout the entire length of the intestine where such obstruction exists, a true necrobiosis of the plexuses of Meissner and Auerbach.

5. In cases of a chronic saturnism, the author observed complete degeneration of the plexuses of the intestine, accompanied with an advanced sclerosis of the semilunar ganglion and of the entire solar plexus.

6. In individuals with chronic catarrh of the intestines, the author observed simple and pigmented atrophy of the plexuses of Meissner and Auerbach. W. C. K.

\section{PARALYSIS FROM LIGHTNING}

Dr. R. V. Limbeck, of Prague, read a paper upon this subject at a recent meeting of the Society of German Physicians of that city.

Lightning can cause paralysis in man and animals, either by laceration of the tissues and hamorrhage, or by direct 
lesion of the nervous and motor apparatus. Holding this view, Mr. Limbeck divides paralysis, due to lightning, into two classes, namely, the false or indirect (dependent upont hemorrhages) and the direct or true varieties. Two cases, which were observed by the speaker at Pribram's Clinic in Prague, corresponded to these types. One case (indirect paralysis) was that of a sixty-seven year old man, who, after being struck with lightning, presented a typical unilateral (right-sided) monoplegia, with motor aphasia. The second case (direct paralysis) was that of a fifty-three-ycar-old trackwalker, who, being struck by lightning, developed a motor and sensory paralysis of his left forearm and hand. The anæsthesia disappeared completely within three days, while the motor paralysis persisted for weeks, In addition to these, the patients presented symptoms which corresponded to the picture of a traumatic neurosis. Amongst these were: Circumscribed anæsthesia of the skin of both feet, bluish discoloration of the skin of both feet, with hyperidrosis, motor (functional) weakness of the right hand, no narrowing of the field of vision.

The writer divides the symptoms of true paralysis from lightning into two groups, namely, those which are directly due to the paralysis and those appearing in the later stages and belonging to a traumatic neurosis. The fact that in the first group of symptoms the sensory paralytic symptoms disappear chiefly more quickly than the motor, the speaker explained by stating that he thinks lightning to more effect the muscle in its function than the peripheral nerves.(Wiener medizinische Wochenschrift, No. 28, I89I.)

$$
\text { F. H. I. }
$$

\section{HYSTERICAL ANOREXIA AND ITS TREATMEÑT.}

Follier, in a recent monograph on the above subject, distinguishes two forms - the one primary, the other secondary.

The primary form " enters into the category of the monosymptomatic manifestations of hysteria." It is the most grave, the most tenacious, and also the most deceptive, for it may be, and it often is, the first manifestation of the hysteria, and is not accompanied by any clear stigmata of the disease. It is particularly in young girls that this form is met; and of all the alarming accidents of this ncurosis. grave anorexia is unquestionably the worst, for it may end in death. What dominates in this affection is not so much the simple want of appetite as the systematic opposition to 